\title{
Evaluation of dosing strategy for pembrolizumab for oncology indications
}

Tomoko Freshwater ${ }^{1}$, Anna Kondic ${ }^{1}$, Malidi Ahamadi ${ }^{1}$, Claire H. Li', Rik de Greef², Dinesh de Alwis ${ }^{1}$ and Julie A. Stone 1* $^{*}$

\begin{abstract}
Background: Traditionally, most monoclonal antibodies (mAbs) have been dosed based on body weight because of perceived contribution of body size in pharmacokinetic variability. The same approach was used in the initial pembrolizumab studies; however, following availability of PK data, the need for weight-based dosing for pembrolizumab was reassessed.
\end{abstract}

Methods: A previously established population PK (popPK) model as well as exposure-response results from patients with advanced melanoma or non-small cell lung cancer (NSCLC) were used to evaluate the potential application of a fixed dosing regimen with the aim of maintaining pembrolizumab exposures within the range demonstrated to provide near maximal efficacy and acceptable safety. Individual PK exposures for the selected fixed dosing regimen from recently completed trials with head and neck cancer, NSCLC, microsatellite instability high (MSI-H) in colorectal cancer (CRC) and urothelial cancer were used to confirm acceptability. To determine whether fixed dosing would maintain exposures within the range of clinical experience, the individual AUC distributions with fixed dosing were compared with the range of exposures from the pembrolizumab doses that were evaluated in early studies (2 $\mathrm{mg} / \mathrm{kg}$ Q3W, 10 mg/kg Q3W/Q2W).

Results: Body-weight dependence of clearance was characterized by a power relationship with an exponent of 0.578 , a value consistent with fixed- and weight-based dosing providing similar control of PK variability. A fixed dose of $200 \mathrm{mg}$ Q3W was investigated in trials based on predicted exposures maintained within the established exposure

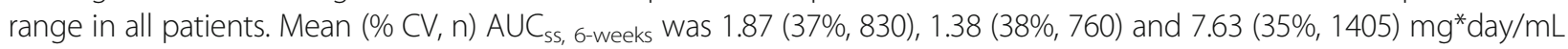
in patients receiving $200 \mathrm{mg}, 2 \mathrm{mg} / \mathrm{kg}$ and $10 \mathrm{mg} / \mathrm{kg}$ Q3W pembrolizumab. High-weight patients had the lowest exposures with $200 \mathrm{mg}$ Q3W; however, exposures in this group (>90 kg) were within the range of prior clinical experience at $2 \mathrm{mg} / \mathrm{kg}$ Q3W associated with near maximal efficacy.

Conclusions: Doses of $200 \mathrm{mg}$ and $2 \mathrm{mg} / \mathrm{kg}$ provide similar exposure distributions with no advantage to either dosing approach with respect to controlling PK variability. These findings suggest that weight-based and fixed-dose regimens are appropriate for pembrolizumab.

Keywords: Pembrolizumab, Fixed dose, Flat dose, Dosing strategy, Clinical dose, Exposure-response analysis, Population pharmacokinetics analysis

\footnotetext{
* Correspondence: julie_stone@merck.com

1Department of Pharmacokinetics, Pharmacodynamics and Drug Metabolism,

Merck \& Co., Inc., 2000 Galloping Hill Road, Kenilworth, NJ, USA

Full list of author information is available at the end of the article
}

\section{Biomed Central}

(c) The Author(s). 2017 Open Access This article is distributed under the terms of the Creative Commons Attribution 4.0 International License (http://creativecommons.org/licenses/by/4.0/), which permits unrestricted use, distribution, and reproduction in any medium, provided you give appropriate credit to the original author(s) and the source, provide a link to the Creative Commons license, and indicate if changes were made. The Creative Commons Public Domain Dedication waiver (http://creativecommons.org/publicdomain/zero/1.0/) applies to the data made available in this article, unless otherwise stated. 


\section{Background}

Dosing of therapeutic monoclonal antibodies (mAbs) is often based on body size, operating from the perception that this reduces intersubject variability in drug exposure [1]. However, because of the specific properties of mAbs (selective mode of action, with substantial therapeutic window) and the advantages of fixed dosing (increased convenience, elimination of wastage, improved safety resulting from a reduced chance for dosing errors, and improved compliance), the weight-based dosing paradigm has recently been re-evaluated [1-3].

Pembrolizumab (Keytruda, Merck \& Co., Inc., Kenilworth, NJ, USA) is a potent, humanized IgG4 monoclonal antibody against programmed death 1 (PD-1) receptor that directly blocks the interaction between PD-1 and its ligands, PD-L1 and PD-L2. Pembrolizumab has demonstrated robust, durable antitumor activity and a manageable safety profile against several advanced malignancies. Early clinical studies of pembrolizumab employed a body-weight-based dosing strategy of $2 \mathrm{mg} / \mathrm{kg}$ every 3 weeks (Q3W) to $10 \mathrm{mg} / \mathrm{kg}$ every 2 weeks (Q2W), but in more recent trials a fixed-dose regimen (fixed with respect to body weight) has been introduced. In this paper, the analyses used to evaluate weightbased vs fixed dosing for pembrolizumab are described and the basis for the decision to switch to fixed-dose regimens in clinical trials is discussed. Results from clinical investigations of $200 \mathrm{mg}$ Q3W are provided as confirming the resulting exposures of the selected fixed dose.

The assessment of the potential to apply a fixed-dose regimen for pembrolizumab used a previously established population pharmacokinetics (popPK) model [4] and therapeutic window information derived from doseresponse and exposure-response results in patients with advanced melanoma or non-small cell lung cancer (NSCLC) enrolled in KEYNOTE-001 [5], KEYNOTE002 [6], and KEYNOTE-006 [7]. The mechanism of action of pembrolizumab, binding to PD-1 receptors on $\mathrm{T}$ cells, does not depend on direct engagement of the molecule with tumor cells. For this reason, substantial differences in exposure-response and doseresponse are not expected across different tumor types. Indeed, it has been found that the pharmacokinetics (PK) of pembrolizumab are similar across oncology indications [4]. On this basis, selection of a fixed-dose regimen focused on establishing a dose that would provide comparable (central tendency and distribution) exposures as the $2 \mathrm{mg} / \mathrm{kg}$ Q3W regimen approved in the United States for melanoma and NSCLC. The fixed dose selected also aimed to maintain exposures within the existing clinical experience range that has been established for melanoma and NSCLC and which has been associated with a lack of clinically important differences in efficacy or safety (Fig. 1).

\section{Methods}

\section{Clinical studies}

Data to inform the fixed-dose evaluations were based on cross-study pooling from a number of ongoing and completed pembrolizumab clinical trials (Table 1). Further description of the trials are in Additional file 1: Table S1 including protocol numbers, title of the protocols and ClinicalTrials.gov identifier, with references to the published primary clinical results paper provided where available. These studies were conducted in accordance with the protocol, good clinical practice standards and the Declaration of Helsinki. The protocols and subsequent amendments were approved by the appropriate institutional review board (IRB) or ethics committee at each participating institution. All patients provided voluntary written informed consent.

The patients were treated with pembrolizumab in a dose range of $1-10 \mathrm{mg} / \mathrm{kg}$ administered as intravenous infusion, with the vast majority of the data collected under 4 dosing regimens (2 mg/kg Q3W, $10 \mathrm{mg} / \mathrm{kg}$ Q3W, $10 \mathrm{mg} / \mathrm{kg}$ Q2W, and $200 \mathrm{mg}$ Q3W) as detailed in Table 1. Data used in this analysis included baseline patient characteristics (demographic factors, measure of renal and hepatic function, and measures of disease severity) and serum concentrations collected from these studies. Most pembrolizumab trials did not collect intensive (serial time-course) PK samples, which would allow for model-independent determination of PK parameter values such as area-under-the-curve (AUC)

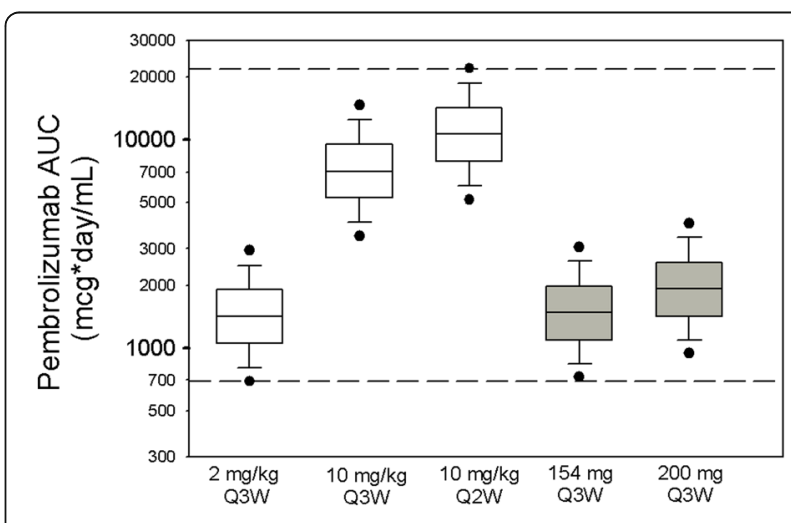

Fig. 1 Simulated distribution of steady-state AUC exposures ( 2800 replicate simulations) for the weight-based regimens of $2 \mathrm{mg} / \mathrm{kg}$ Q3W, $10 \mathrm{mg} / \mathrm{kg} \mathrm{Q} 3 \mathrm{~W}$, and $10 \mathrm{mg} / \mathrm{kg}$ Q2W compared with the simulated distribution of exposures for two potential fixed-dose regimens (log scale): Box: straight middle line= median; edges $=$ 25th and 75th percentiles; whiskers $=10$ th and 90th percentiles; dots $=5$ th and 95th percentiles. Horizontal dashed lines represent the range of exposures (5th percentile of $2 \mathrm{mg} / \mathrm{kg}$ Q3W and 95th percentile of $10 \mathrm{mg} / \mathrm{kg}$ Q2W) from dose regimens demonstrated to have comparable efficacy and tolerability in melanoma and NSCLC trials 
Table 1 Number of patients with observed PK concentration data used in the analysis by study, cancer type and dosing regimen

\begin{tabular}{|c|c|c|c|c|c|}
\hline Study & Cancer Type & $2 \mathrm{mg} / \mathrm{kg}$ Q3W & 10 mg/kg Q3W & 10 mg/kg Q2W & $200 \mathrm{mg}$ Q3W \\
\hline KEYNOTE-001 & Melanoma & 165 & 309 & 176 & \\
\hline KEYNOTE-001 & NSCLC & 61 & 288 & 204 & \\
\hline KEYNOTE-002 & Melanoma & 207 & 212 & & \\
\hline KEYNOTE-006 & Melanoma & & 270 & 272 & \\
\hline KEYNOTE-010 & NSCLC & 327 & 326 & & \\
\hline KEYNOTE-024 & NSCLC & & & & 152 \\
\hline KEYNOTE-045 & Urothelial Cancer & & & & 262 \\
\hline KEYNOTE-052 & Urothelial Cancer & & & & 311 \\
\hline KEYNOTE-055 & HNSCC & & & & 47 \\
\hline KEYNOTE-164 & MSI-H & & & & 58 \\
\hline
\end{tabular}

There are patients with missing indication for $N=24$ from KEYNOTE-001, 002 and 006

NSCLC Non-Small Cell Lung Cancer, HNSCC Head and Neck Squamous Cell Carcinoma, MSI-H Microsatellite Instability-High Carcinoma

on a given study day. Rather sparse PK samples (1 or 2 samples per designated clinic visit) have been collected to minimize the burden on patients. The available concentration data were obtained either at peak (nominally within $30 \mathrm{~min}$ after end of infusion) or trough (nominally within $24 \mathrm{~h}$ before the next dose) samples and were obtained during pre-specified dosing cycles throughout the pembrolizumab treatment. Actual time of dosing and PK sampling were collected and used in the analyses. PK samples for pembrolizumab serum concentration determination were assayed using previously reported methods [4].

\section{Pharmacokinetics analysis}

PopPK analysis was used to estimate PK parameters and exposures from observed sparse concentration data and to simulate PK under potential fixed dosing regimens, which informed the fixed dose selected for investigation in the trials. PopPK is a model-based approach to describe the time course of drug exposure across individuals in a population by estimation of both population-level typical PK values (eg, clearance, volume of distribution) and explicit terms to describe variability, including inter-subject variability, underlying the distribution of responses. It is the preferred method for interpreting sparse PK concentration data $[8,9]$.

A two-compartment pembrolizumab popPK model was used that was previously established based on data from KEYNOTE-001, -002 and -006 and is described in detail in [4]. The relationships between PK parameters (clearance $[\mathrm{CL}]$ and volume of distribution $[\mathrm{Vc}]$ ) and body weight were estimated by the incorporation of an allometric exponential relationship with body-weight in the terms for these parameters:

$$
\begin{aligned}
& C L=C L_{T V} \cdot\left(\frac{W T}{\operatorname{Median}(W T)}\right)^{\alpha-C L} \cdot e^{\eta_{1}} \\
& V c=V c_{T V} \cdot\left(\frac{W T}{\operatorname{Median}(W T)}\right)^{\alpha-V c} \cdot e^{\eta_{2}}
\end{aligned}
$$

where $X_{T V}$ is the typical value of the pharmacokinetic parameter $\mathrm{X}$, and $\alpha-\mathrm{X}$ is the allometric exponent describing the association with WT (individual body weight) normalized by MedianWT. The terms $\mathrm{e}^{\eta} \mathrm{de}$ scribed further inter-individual variation in these PK parameters beyond that accounted for by WT. Two additional parameters $\left(\mathrm{Q}\right.$ and $\left.\mathrm{V}_{\mathrm{P}}\right)$ described the distribution behavior of pembrolizumab and were also adjusted for WT, using the same values for the exponents as for $\mathrm{CL}$ and $\mathrm{Vc}$, respectively. Covariate terms describing other factor associations were retained in the popPK model as identified in [4], and included sex, baseline estimated glomerular filtration rate (eGFR), baseline albumin, prior treatment with ipilimumab, cancer type, baseline Eastern Cooperative Oncology Group (ECOG) performance status, and baseline tumor burden (sum of longest dimensions of target lesions).

PK Estimation: The popPK model was re-estimated by fitting the previous dataset with the new KEYNOTE-10, KEYNOTE-055, KEYNOTE-024, KEYNOTE-164, KEYNOTE-045 and KEYNOTE-052 concentration data added to obtain individual post-hoc PK parameter estimates from which individual PK values were derived for AUC at steady state, over 6 weeks $\left(\mathrm{AUC}_{\mathrm{ss}}, 0-6\right.$ weeks $)$, steady-state peak serum concentration $\left(C_{\max }\right.$, ss $)$ and steady-state trough serum concentration $\left(C_{\text {trough, ss }}\right)$. $\mathrm{AUC}_{\mathrm{ss}}$, 0-6weeks was calculated as: 


$$
A U C=\frac{\operatorname{Actualdose}(m g)^{*}}{\text { Clearance }\left(\frac{L}{\text { day }}\right)} \times \frac{6(\text { weeks })}{\text { dosing interval }(\text { weeks })}
$$

$\mathrm{C}_{\max }$, ss and $\mathrm{C}_{\text {trough, ss }}$ were determined from the concentration-time profile using each individual's posthoc estimated pharmacokinetic parameters. Summary statistics (mean, \%CV, median, 10-90 percentiles) were determined by using $\mathrm{R}$ version 3.2.5 (Free Software Foundation, Boston, MA).

PK Simulation: To predict pembrolizumab PK under untested dosing regimens, virtual oncology patients were created by randomly drawing covariate values (body weight, albumin, bilirubin, baseline tumor burden, estimated glomerular filtration rate, sex, tumor type, baseline ECOG performance status, ipilimumab history) with replacement from the pooled baseline covariate data from available pembrolizumab studies $(n=3038$ from KEYNOTE-001, -002, -006, -010, -011, -012, -025, -041 and -055) (Additional file 1: Table S1) to enable the simulation of exposures using the popPK model (2800 virtual patients simulated per each dosing group). Intersubject variability terms in the model were sampled from the established distributions, which together with fixed parameters (typical values and covariate relationships) determined parameter values (eg, CL, Vc) for each virtual patient, which were in turn used to determine PK values $\left(\mathrm{AUC}_{\mathrm{ss}}, 0\right.$ 0-6weeks $\left., \mathrm{C}_{\mathrm{max}, \mathrm{ss}}, \mathrm{C}_{\text {trough, ss }}\right)$ as described above. Graphical plots were generated using R version 3.2.5 (Free Software Foundation, Boston, MA) and SigmaPlot 11.0 (Systat Software Inc., San Jose, CA).

\section{Drug product wastage calculation}

To quantify the impact of a fixed-dosing regimen on drug product usage, the amount of remaining pembrolizumab product per single drug administration at $2 \mathrm{mg} / \mathrm{kg}$ Q3W using currently available 50 - or $100-\mathrm{mg}$ vials was calculated. First, the weight distribution in a typical oncology population was generated for 1000 subjects at random from the observed weight distribution in the popPK dataset $(3.7 \%$ of $\leq 50 \mathrm{~kg}, 31.9 \%$ of $>50-\leq 70 \mathrm{~kg}, 39.4 \%$ of $>70-\leq 90 \mathrm{~kg}, 22.3 \%$ of $>90-\leq 120 \mathrm{~kg}$ and $2.7 \%$ of $>120 \mathrm{~kg}$ : Additional file 2: Table S2). The total amount of dose in $\mathrm{mg}$ for each subject was derived as the product of $2 \mathrm{mg} / \mathrm{kg}$ and body weight. Based on the total dose amount, the number of vials required was determined based on $50-\mathrm{mg}$ or $100-\mathrm{mg}$ vials available. The amount of remaining product for each subject per $2 \mathrm{mg} / \mathrm{kg}$ administration was calculated by the difference in the total amount available in the required vials and the total amount needed to dose at $2 \mathrm{mg} / \mathrm{kg}$. Individual subject results were binned into 5 groups $(0-10 \mathrm{mg}, 10-20 \mathrm{mg}$, 20-30 mg, 30-40 mg, 40-50 mg for 50-mg vial, and
0-20 mg, 20-40 mg, 40-60 mg, 60-80 mg, 80$100 \mathrm{mg}$ for $100-\mathrm{mg}$ vial). Additionally, the overall amount of remaining drug product resulting from a full treatment course in these 1000 subjects was estimated by summing the product of the individual estimates for remaining product per dosing event and the typical number of doses received in a treatment course given the average pembrolizumab treatment duration of 6.2 months ( $~ 8$ doses) (based on experience in early melanoma trials as described in label issued in September 2014).

\section{Results}

\section{PopPK model and effect of body weight}

Body weight is a known factor in drug exposure of therapeutic antibodies and therefore the weight-dependency of the PK properties was robustly evaluated in the popPK modeling efforts. The dataset used in a previously reported popPK analysis included 1622 patients (73.9\%) with advanced melanoma, 551 patients (25.1\%) with advanced NSCLC, and 22 patients (1.0\%) with other advanced malignancies [4] from KEYNOTE-001, -002 and -006. The analysis population encompassed a wide distribution of body weight, with a median of $77.2 \mathrm{~kg}$ and a range of $35.7-209.5 \mathrm{~kg}$. Estimates (90\% confidence intervals [CI]) of the relationship between clearance and body weight based on the popPK model revealed an allometric exponent $(\alpha)$ of 0.578 (95\% CI, 0.481-0.666) for the clearance parameters and $0.492(95 \% \mathrm{CI}, 0.432-0.553)$ for the volumes of distribution. Theoretically, fixed dosing would work the best when $\mathrm{CL}$ is not affected by body weight $(\alpha=0)$ and body-weight-based dosing would work the best when $\alpha$ equals to 1 [1-3]. Given that $\alpha$ estimates were close to 0.5 for both clearance and volume of distribution, no advantage of weight-based dosing over fixed dosing is expected, and both weight-based and fixed dosing should provide adequate and similar control of PK variability.

\section{Simulation results informing selection of $200 \mathrm{mg}$ Q3W as fixed dose for investigation in trials}

The expected distributions of pembrolizumab exposures from potential fixed doses administered Q3W were simulated using the popPK model and compared with the distributions expected from the weight-based dosing regimens $(2 \mathrm{mg} / \mathrm{kg}$ Q $3 W, 10 \mathrm{mg} / \mathrm{kg}$ Q3W, and $10 \mathrm{mg} / \mathrm{kg}$ Q2W) studied in the melanoma and NSCLC trials that supported the current US registrations. The distribution of exposures from these weight-based regimens represents the range of clinical experience to date, where the safety profile, overall response rate and survival outcomes have been found to be similar across the tested dosing regimens in the melanoma and NSCLC trials $[5-7,10,11]$, and a flat exposure-response relationship 
was identified across these three regimens in evaluations of tumor size response $[12,13]$ and immune-related adverse events [6, 7]. Based on this flat dose- and exposureresponse relationship, $2 \mathrm{mg} / \mathrm{kg}$ Q3W regimen was initially approved in the United States for melanoma and NSCLC as a dosing regimen which achieves clinically meaningful efficacy with limited additional clinical benefit at higher dose levels. Figure 1 displays the distribution of steadystate AUC exposures predicted by the popPK model. The AUC values are displayed on a log scale, allowing for ready comparison of the relative PK variability with weightbased versus fixed dosing. As expected based on the allometric exponent values discussed above, the PK variability from weight-based and fixed-dose regimens is nearly identical. A fixed dosage of $154 \mathrm{mg}$ Q3W was identified as providing almost identical steady-state AUC exposure as a weight-based dosage of $2 \mathrm{mg} / \mathrm{kg}$ Q3W. The distribution of exposures from $200 \mathrm{mg}$ Q3W substantially overlaps that obtained with the $2 \mathrm{mg} / \mathrm{kg}$ Q3W dose and is well within the exposure range associated with maximal clinical response and acceptable tolerability in melanoma and NSCLC.

The predicted variation in pembrolizumab AUC exposure with patient body weight for the $2 \mathrm{mg} / \mathrm{kg}$ Q3W and $200 \mathrm{mg}$ Q3W regimens is shown in Fig. 2. For the weight-based regimen, lower-weight patients tend to have lower exposures relative to higher-weight patients, while the opposite trend is seen with fixed dosing. For both regimens, the range of individual exposures for the low-weight patients considerably overlaps that for the high-weight patients, consistent with PK variability being only partially explained by weight. The overall extent of PK variability appears similar for both regimens. The $200 \mathrm{mg}$ Q3W regimen was selected for investigation in the clinical trials based on the similarity of the exposures to $2 \mathrm{mg} / \mathrm{kg}$ with a slight upward shift to ensure individual patient exposures, especially in patients with a higher weight, fall within the range of prior clinical experience.
Of note, with the $200 \mathrm{mg}$ Q3W regimen, very few simulated individual patients fell below the fifth percentile of exposures from the approved $2 \mathrm{mg} / \mathrm{kg}$ Q3W regimen. Both regimens were predicted to yield a range of exposures that falls well below the highest exposure for which acceptable tolerability has been demonstrated.

\section{Observed $200 \mathrm{mg}$ Q3W fixed-dose exposures}

Observed PK data for $200 \mathrm{mg}$ Q3W fixed dosing from patients with head and neck cancer, NSCLC, MSI-H in $\mathrm{CRC}$ and urothelial cancer treated with pembrolizumab in KEYNOTE-055, -024, -164, -52 and -045, respectively, confirm the exposure predicted for this regimen based on the popPK model. The observed concentration data from $200 \mathrm{mg}$ Q3W are consistent with the modelpredicted time course of concentration over the dosing interval both early in therapy and after PK steady-state is achieved (Fig. 3). Figure 3 also illustrates that the shape of the PK concentration-time profile with the fixed-dose regimen is similar to that obtained with the $2 \mathrm{mg} / \mathrm{kg}$ regimen in the earlier trials. The AUC exposures obtained in the $200 \mathrm{mg}$ Q3W trials also indicate a good match of observed and predicted PK, with the distribution of observed exposures falling within the range of previous clinical experience derived from the weightbased regimens (Fig. 4a). In this analysis, PK data were obtained in patients with several cancer types not previously described. Clearance values across all cancer types were not meaningfully different (Fig. 5), supporting the consistency of pembrolizumab PK across cancer types.

Summary statistics for the observed pembrolizumab exposures across the 4 dosing regimens (Table 2) indicate that the central tendency (mean, median) at $200 \mathrm{mg}$ Q3W is modestly increased ( $35 \%)$ relative to $2 \mathrm{mg} / \mathrm{kg}$ $\mathrm{Q} 3 W$ for all PK measures $\left(\mathrm{AUC}_{\mathrm{ss}}, 0\right.$-6weeks, $\mathrm{C}_{\mathrm{max} \text {, ss }}$ and $\mathrm{C}_{\text {trough, ss }}$ ), while these values are $\sim 25 \%$ of those obtained at $10 \mathrm{mg} / \mathrm{kg}$ Q3W. Intersubject variation (\% CV) is
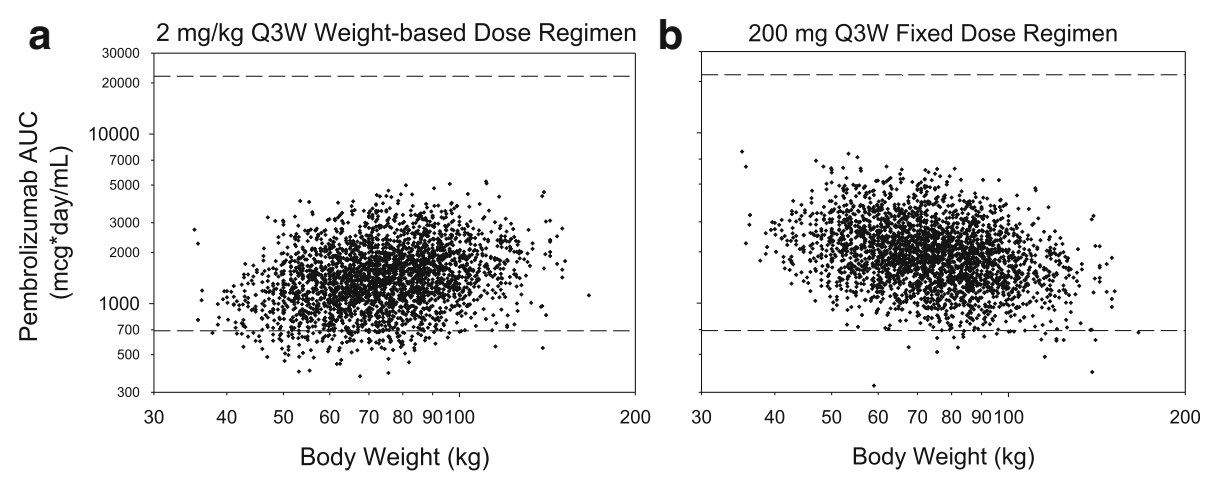

Fig. 2 Predicted variation in pembrolizumab AUC exposure by body weight for weight-based (a) and fixed-dose (b) regimens (2800 replicate simulations): Horizontal dashed lines represent the range of exposures (5th percentile of $2 \mathrm{mg} / \mathrm{kg}$ Q3W and 95th percentile of $10 \mathrm{mg} / \mathrm{kg}$ Q2W) from dose regimens demonstrated to have comparable efficacy and tolerability in melanoma and NSCLC trials 


\section{First Dose 2 mg/kg}

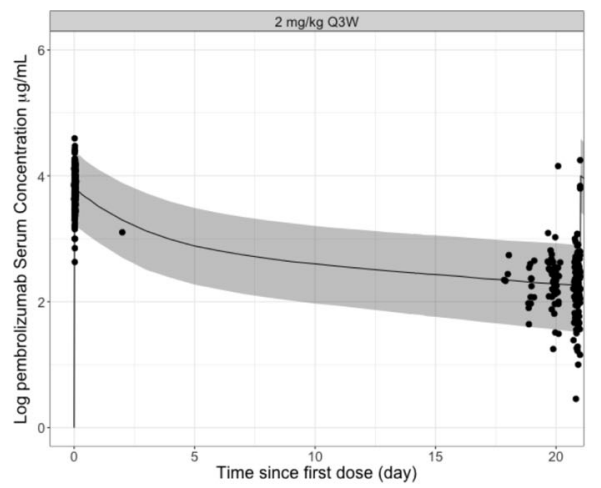

First Dose 200 mg

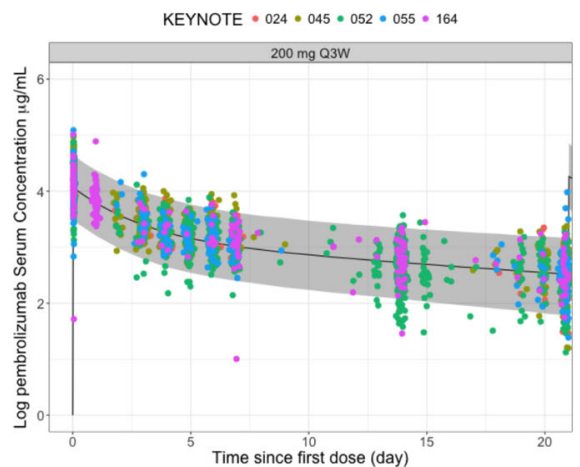

\section{Steady-State $2 \mathrm{mg} / \mathrm{kg}$}

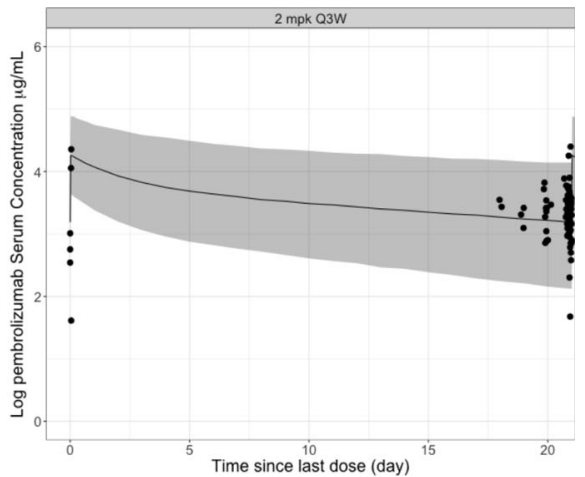

Steady-State $200 \mathrm{mg}$

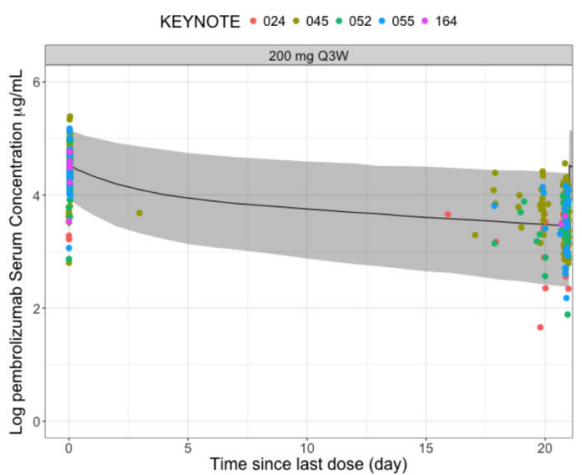

Fig. 3 Consistency of observed concentrations in patients with predictions based on population PK model: Pembrolizumab concentration-time profiles during the first dose (left pane/s) and at steady state (right panels) of repeated dosing at $2 \mathrm{mg} / \mathrm{kg}$ Q3W (top panels) and $200 \mathrm{mg}$ Q3W (bottom panels). Solid markers represent observed pembrolizumab serum concentrations. Solid line represents median predicted concentration time profile, based on the population PK model. Shaded areas represent 90\% prediction interval for the prediction
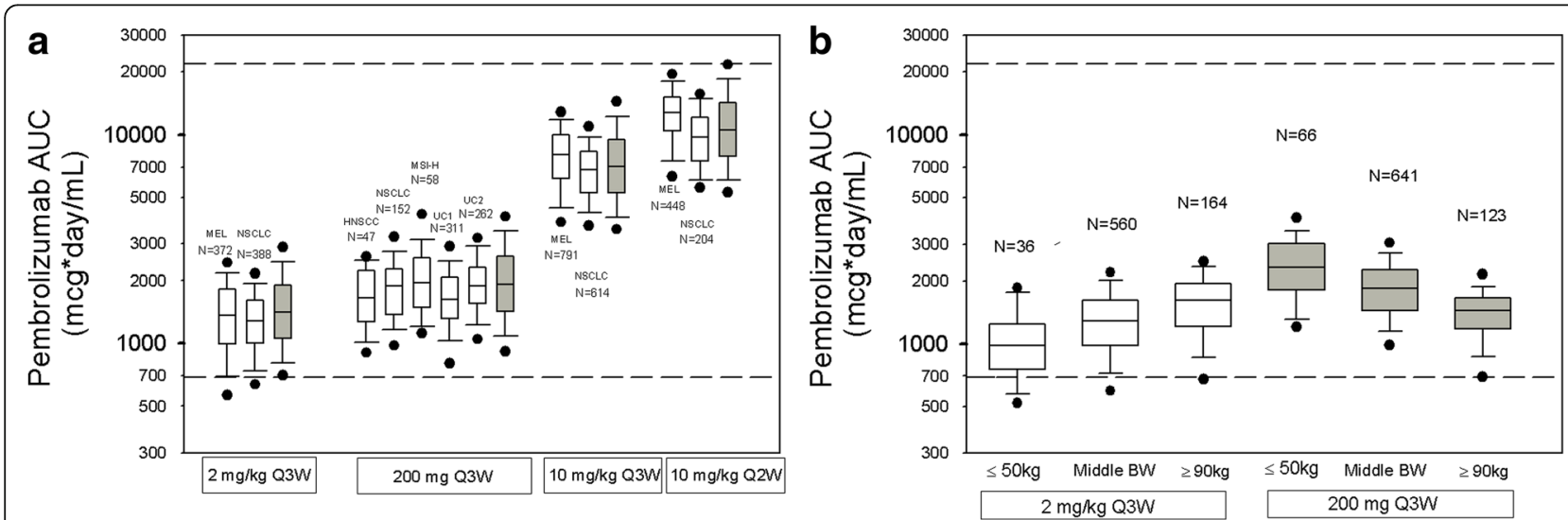

Fig. 4 Distribution of observed pembrolizumab $A_{U C} C_{5 s}$ 0-6weeks: Panel a - Consistency with model predictions (Simulated values shown in gray and observed values in white). Panel $\mathbf{b}$ - Variation in exposures with body weight under weight-based versus fixed dosing. Box: straight middle line $=$ median; edges $=25$ th and 75 th percentiles; whiskers $=10$ th and 90th percentiles; dots $=5$ th and 95th percentiles. Horizontal dashed lines (__ _ - represent the range of exposures (5th percentile of $2 \mathrm{mg} / \mathrm{kg}$ Q3W and 95th percentile of $10 \mathrm{mg} / \mathrm{kg}$ Q2W) from dose regimens demonstrated to have comparable efficacy and tolerability in melanoma and previously treated NSCLC trials. Observed data are based on Table 1. In Panel B, distribution of observed $A \cup C_{55}$ 0-6weeks for light $(\leq 50 \mathrm{~kg}$ ), middle (between 50 and $90 \mathrm{~kg}$ ) and heavy ( $\geq 90 \mathrm{~kg}$ ) body-weight patients 


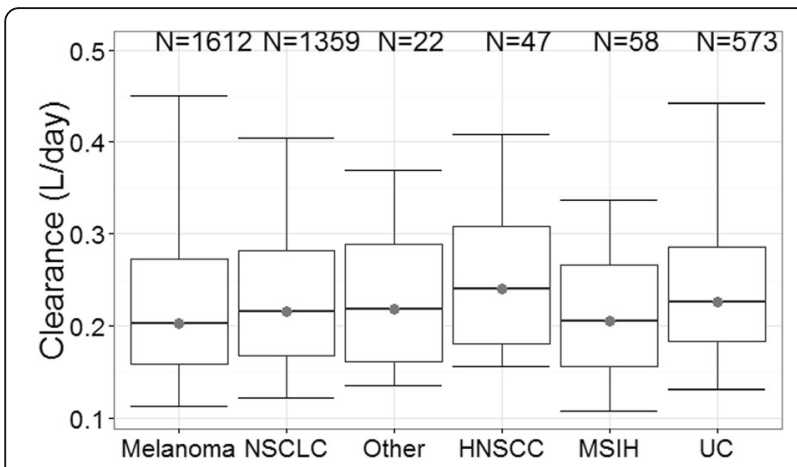

Fig. 5 Consistency of pembrolizumab clearance in patients with differing cancer: melanoma from KEYNOTE-001, -002 and -006. NSCLC from KEYNOTE-001,-010 and -024. Other (other cancers) from KEYNOTE-001 in initial cohort. HNSCC (head and neck trial) from KEYNOTE-055. MSIH (MSI-H in CRC) from KEYNOTE-164. UC (urothelial cancer trial) from KEYNOTE-045 and -052

similar for all regimens and the $10-90 \%$ percentiles are largely overlapping for $2 \mathrm{mg} / \mathrm{kg}$ and $200 \mathrm{mg}$ Q3W. The distribution of observed exposures with the $2 \mathrm{mg} / \mathrm{kg}$ and $200 \mathrm{mg}$ Q3W regimens were compared among three weight-based subpopulations: light (body weight $\leq 50 \mathrm{~kg}$ ), middle (body weight between 50 and $90 \mathrm{~kg}$ ) and heavy (body weight $\geq 90 \mathrm{~kg}$ ) to investigate the influence of extreme body weights on exposures (Fig. 4b). The distribution of body weights in the patients studied under these two regimens was similar (Additional file 3: Figure S1). The influence of body weight trended as predicted in the simulations. Although heavier patients had lower exposures with the $200 \mathrm{mg}$ fixed dose, the distribution of exposures obtained in these patients was contained within the range of exposures from the prior clinical experience.

\section{Discarded amount with weight-based dosing}

The amount of remaining pembrolizumab product from administration of a $2 \mathrm{mg} / \mathrm{kg}$ weight-based dose using 50or 100-mg vials was estimated based on the distribution of body weight in the analysis dataset (Additional file 4: Table S3). On average, 27 and $56 \mathrm{mg}$ per patient per administration would remain when using 50- and 100-mg vials, respectively. Given that the average pembrolizumab treatment duration was 6.2 months (approximately eight doses) in patients with melanoma treated in KEYNOTE-001, approximately 220 g or 450 g pembrolizumab would be remaining for every 1000 patients treated using the weight-based dosing with the 50- or 100-mg vials, respectively. By contrast, no remaining product is expected at 200-mg fixed dosing using 50- or 100 -mg vials.

\section{Discussion}

The evaluations and illustrations provided in this paper provide an example that for mAbs, there is no set answer as to whether weight-based or fixed-dose strategies are better. Therefore, the appropriate dosing strategy should be evaluated based on the PK properties of the given mAb. It was demonstrated in this paper that both weight-based and fixed dosing are appropriate for pembrolizumab, with neither regimen providing a PK advantage over the other. Although there is no PK advantage for either regimen, fixed dosing would eliminate the waste generated by weight-based dosing, improve compliance and might also reduce the risk of dosing errors by reducing dosing complexity.

Pembrolizumab is currently available in 50- or 100-mg vials. When using a weight-based dosing regimen, the contents of the final vial are generally incompletely

Table 2 Pharmacokinetics of pembrolizumab at steady state of regimens of 2 mg/kg Q3W, 200 mg Q3W, 10 mg/kg Q3W and $10 \mathrm{mg} / \mathrm{kg}$ Q2W. Based on pooled cross-study data [n, Mean (\%CV), Median (10-90 percentile)]

\begin{tabular}{|c|c|c|c|c|c|}
\hline PK Value (unit) & Dose Regimen & $\mathrm{N}$ & Mean $(\% \mathrm{CV})$ & Median & 10-90 Percentile \\
\hline \multirow{4}{*}{$\begin{array}{l}\mathrm{C}_{\max } \\
(\mathrm{mcg} / \mathrm{mL})\end{array}$} & $2 \mathrm{mg} / \mathrm{kg} \mathrm{Q3W}$ & 755 & $68.0(24 \%)$ & 66.3 & $48.3-88.2$ \\
\hline & 200 mg Q3W & 830 & $93.4(26 \%)$ & 89.1 & $66.4-124.3$ \\
\hline & 10 mg/kg Q3W & 1403 & $360.3(23 \%)$ & 357.6 & $257.7-466.8$ \\
\hline & 10 mg/kg Q2W & 652 & $459.3(25 \%)$ & 457.7 & $315.9-599.9$ \\
\hline \multirow{4}{*}{$\begin{array}{l}\text { C } \text { trough } \\
(\mathrm{mcg} / \mathrm{mL})\end{array}$} & $2 \mathrm{mg} / \mathrm{kg} \mathrm{Q3W}$ & 755 & $22.2(48 \%)$ & 21.1 & $9.18-35.7$ \\
\hline & 200 mg Q3W & 830 & $29.7(47 \%)$ & 27.6 & $14.9-46.2$ \\
\hline & 10 mg/kg Q3W & 1403 & $126.4(44 \%)$ & 120.4 & $59.8-200.2$ \\
\hline & 10 mg/kg Q2W & 652 & 220.9 (39\%) & 217.8 & $111.8-325.3$ \\
\hline \multirow{4}{*}{$\begin{array}{l}\mathrm{AUC}_{\mathrm{ss},}, 0-6 \text {-weeks } \\
\left(\mathrm{mcg}^{*} \text { day } / \mathrm{mL}\right)\end{array}$} & $2 \mathrm{mg} / \mathrm{kg}$ Q3W & 760 & $1376.5(38 \%)$ & 1316.5 & 724.9-2038.5 \\
\hline & 200 mg Q3W & 830 & $1871.1(37 \%)$ & 1787.0 & $1120.6-2730.9$ \\
\hline & 10 mg/kg Q3W & 1405 & 7625.4 (35\%) & 7436.0 & $4354.0-11172.8$ \\
\hline & 10 mg/kg Q2W & 652 & 12002.7 (34\%) & 11993.5 & 6834.7-16895.5 \\
\hline
\end{tabular}


administered, and the remaining drug product is discarded as per labeling instructions. In practice it might potentially be used for another patient, raising quality concerns and, consequently, potential safety concerns as it represents a source of infection when it is used inappropriately outside of the clinical trial setting. Centers for Disease Control and Prevention (CDC) reported several instances including mishandling of injectable medications such as reuse of single-dose vials for more than one patient. From 2010-2014, CDC is aware of at least 26 outbreaks due to unsafe injection practices. These outbreaks resulted in more than 95,000 patients being referred for testing after potential exposure to infectious diseases. $73 \%(n=19)$ of these outbreaks involved use of single-dose/single-use medications for more than one patient $[14,15]$.

The results presented in this paper demonstrate that fixed dosing of pembrolizumab $200 \mathrm{mg}$ Q3W maintains exposures comparable with or slightly increased relative to those from $2 \mathrm{mg} / \mathrm{kg}$ Q3W (the initially approved dose for pembrolizumab). All patients, including high-weight patients, achieve exposures in the range which has been demonstrated in clinical dose-ranging trials to provide near maximal efficacy. Exposures achieved by $200 \mathrm{mg}$ Q3W also fall well below the high dose clinical experience at $10 \mathrm{mg} / \mathrm{kg}$ for which acceptable tolerability has been demonstrated. Exposures which match or exceed those at $2 \mathrm{mg} / \mathrm{kg}$ also ensure that maximal target engagement is achieved as informed by early PK/PD work with a clinical biomarker (IL-2 release) in KEYNOTE-001 which demonstrated saturation of response at $1 \mathrm{mg} / \mathrm{kg}[16,17]$. The estimated median clearance of pembrolizumab in the patients receiving $200 \mathrm{mg}$ Q3W was $0.22 \mathrm{~L} /$ day, which is similar to the $0.23 \mathrm{~L} /$ day obtained in patients receiving $2 \mathrm{mg} / \mathrm{kg}$ Q3W as well as the clearance of endogenous IgG $(0.21 \mathrm{~L} /$ day $)$ and consistent with linear clearance characteristics of typical mAbs $(0.2-0.5 \mathrm{~L} /$ day $)[18,19]$. The consistency of PK across cancer types supports the use $200 \mathrm{mg}$ in Q3W in various cancer types.

Recently, $200 \mathrm{mg}$ Q3W was approved for use in patients with NSCLC [20] and HNSCC [21-23] in the United States, and clinical results show similar efficacy and safety in these indications among doses $(2 \mathrm{mg} / \mathrm{kg}$ Q3W, $200 \mathrm{mg}$ Q3W, $10 \mathrm{mg} / \mathrm{kg}$ Q3W/Q2W) in the trials supporting these indications.

\section{Conclusions}

In conclusion, the $200 \mathrm{mg}$ Q3W fixed dosage can be considered an appropriate fixed-dose regimen for pembrolizumab based on the achievement of exposures well within the prior clinical experience demonstrated to be associated with near maximal efficacy and acceptable tolerability. The $200 \mathrm{mg}$ Q3W dose of pembrolizumab, which continues to be investigated in trials for various oncology indications, may also be an appropriate alternative for patients currently being treated with the approved $2 \mathrm{mg} / \mathrm{kg}$ Q3W dose.

\section{Additional files}

Additional file 1:Table S1. Description of clinical trials used in analyses [24]. (DOCX $13 \mathrm{~kb})$

Additional file 2: Table S2. Body weight distribution in the population PK analysis dataset ( $N=2195 ;$ KEYNOTE-001 + KEYNOTE-002 + KEYNOTE-006). (DOCX $12 \mathrm{~kb}$ )

Additional file 3: Figure S1. Observed body weight distribution for $2 \mathrm{mg} / \mathrm{kg}$ Q3W and $200 \mathrm{mg}$ Q3W. Observed weight distribution of total $N=$ 1591 ( $N=760$ who received $2 \mathrm{mg} / \mathrm{kg}$ Q3W and $N=830$ who received $200 \mathrm{mg}$ Q3W). KEYNOTE-001, -002, -010 at $2 \mathrm{mg} / \mathrm{kg}$ Q3W, -024, -052, -055, -045 and -164 at $200 \mathrm{mg}$ Q3W (KEYNOTE-006 contains only $10 \mathrm{mg} / \mathrm{kg}$ ). Median weights: $74.0 \mathrm{~kg}$ for $2 \mathrm{mg} / \mathrm{kg}$ Q3W (solid red line) and $71.8 \mathrm{~kg}$ for 200 mg Q3W (solid blue line). Black dot lines: $50 \mathrm{~kg}$ and 90 kg. (PNG 6 kb)

Additional file 4: Table S3. Distribution of patients as a function of amount (mg) remaining product per one administration at $2 \mathrm{mg} / \mathrm{kg}$ using 50-mg or 100-mg vial. The amount of remaining product was categorized into 5 groups (0-10 mg, 10-20 mg, 20-30 mg, 30-40 mg, 40$50 \mathrm{mg}$ for 50-mg vial, and 0-20 mg, 20-40 mg, 40-60 mg, 60-80 mg, $80-100 \mathrm{mg}$ for $100-\mathrm{mg}$ vial) and the distribution of patients by these categories and the total amount of remaining drug product associated with weight-based dosing. Approximately $20 \%$ of the population would fall in each category. (DOCX $13 \mathrm{~kb}$ )

\section{Abbreviations}

$\%$ CV: Coefficient of variation of between-subject variability of parameters; a: Allometric exponent; AUC: Area-under-the-curve; AUCss: 0-6-weeks, Area under the concentration-time curve at steady state over a 6-week interval; CDC: Centers for Disease Control and Prevention; Cl: Confidence interval;

$\mathrm{CL}$ : Clearance; $C_{\max }$ ss: Steady-state maximum observed serum concentration; CRC: Colorectal cancer; $C_{\text {trough, ss: }}$ Steady-state concentration at the end of the dosing interval; EGFR: Epidermal growth factor receptor; eGFR: Estimated glomerular filtration rate; HNSCC: Head and neck squamous cell carcinoma; IgG: Immunoglobulin G; IL-2: Interleukin-2; IRB: Institutional review board; mAbs: Monoclonal antibodies; MEL: Melanoma; MSI-H: Microsatellite Instability High; N: Number; NSCLC: Non-small cell lung cancer; PD1: Programmed death 1; PD-L1: Programmed death ligand 1;

PK: Pharmacokinetics; popPK: Population pharmacokinetic;

Q: Intercompartmental clearance; Q2W: Every 2 weeks; Q3W: Every 3 weeks; UC: Urothelial cancer; Vc: Central volume of distribution; Vp: Peripheral volume of distribution

\section{Acknowledgements}

We thank Marie-Jose van Lierop, Mariëlle van Zutphen-van Geffen, Jeroen Elassaiss-Schaap, Kapil Mayawala, Manash Chatterjee and David C. Turner for critical review and support of the manuscript. Editorial support in the preparation of this manuscript was provided by Chris Ontiveros, PhD (ApotheCom, New York, NY), and was funded by Merck \& Co., Inc. (Kenilworth, NJ, USA).

\section{Funding}

Financial support for all of the trials discussed in this manuscript was provided by Merck \& Co., Inc. (Kenilworth, NJ, USA).

\section{Availability of data and materials}

The datasets used and/or analyzed during the current study are available from the corresponding author on reasonable request.

\section{Authors' contributions}

TF: Conception, design or planning of the study, acquisition and analysis of the data, interpretation of the results and drafting of the manuscript. AK: Analysis of the data, interpretation of the results and drafting of the manuscript. MA: Analysis of the data and interpretation of the results. CHL: Interpretation of the results. RdeG: Analysis of the data and interpretation of 
the results. DdeA: Interpretation of the results. JAS: Conception, design or planning of the study, analysis of the data and interpretation of the results. All authors critically reviewed iterations of the manuscript and approved the final draft for submission.

\section{Competing interests}

Not applicable.

\section{Consent for publication}

Not applicable.

Ethics approval and consent to participate

Not applicable.

\section{Publisher's Note}

Springer Nature remains neutral with regard to jurisdictional claims in published maps and institutional affiliations.

\section{Author details}

${ }^{1}$ Department of Pharmacokinetics, Pharmacodynamics and Drug Metabolism Merck \& Co., Inc., 2000 Galloping Hill Road, Kenilworth, NJ, USA. ${ }^{2}$ Quantitative Solutions, a Certara Company, Kloosterstraat 9, Oss, The Netherlands.

\section{Received: 15 September 2016 Accepted: 26 April 2017}

Published online: 16 May 2017

\section{References}

1. Bai $S$, Jorga $K$, Xin $Y$, Jin D, Zheng $Y$, Damico-Beyer LA, et al. A guide to rational dosing of monoclonal antibodies. Clin Pharmacokinet. 2012;51(2): $119-35$.

2. Wang DD, Zhang S, Zhao H, Men AY, Parivar K. Fixed dosing versus body size-based dosing of monoclonal antibodies in adult clinical trials. J Clin Pharmacol. 2009;49(9):1012-24.

3. Zhang S, Shi R, Li C, Parivar K, Wang DD. Fixed dosing versus body sizebased dosing of therapeutic peptides and proteins in adults. J Clin Pharmacol. 2012:52(1):18-28.

4. Ahamadi M, Freshwater T, Prohn M, Li CH, De Alwis DP, De Greef R, et al. Model-based characterization of the pharmacokinetics of pembrolizumab, a humanized anti-Pd-1 monoclonal antibody, in advanced solid tumors. CPT Pharmacometrics Syst Pharmacol. 2017;6(1):49-57. doi:10.1002/Psp4.12139. Epub 2016 Nov 14.

5. Ribas A, Hamid O, Daud A, Hodi FS, Wolchok JD, Kefford R, et al. Association of pembrolizumab with tumor response and survival among patients with advanced melanoma. JAMA. 2016;315(15):1600-9.

6. Ribas A, Puzanov I, Dummer R, Schadendorf D, Hamid O, Robert C, et al. Pembrolizumab versus investigator-choice chemotherapy for ipilimumabrefractory melanoma (KEYNOTE-002): a randomised, controlled, phase 2 trial. Lancet Oncol. 2015;16(8):908-18.

7. Robert C, Schachter J, Long GV, Arance A, Grob JJ, Mortier L, et al. Pembrolizumab versus ipilimumab in advanced melanoma. N Engl I Med. 2015;372(26):2521-32.

8. Sheiner LB. The population approach to pharmacokinetic data analysis: rationale and standard data analysis methods. Drug Metab Rev. 1984;15:153-71.

9. Duffull SB, Wright DF, Winter HR. Interpreting population pharmacokineticpharmacodynamic analyses - a clinical viewpoint. Br J Clin Pharmacol. 2011;71(6):807-14

10. Garon EB, Rizvi NA, Hui R, Leighl N, Balmanoukian AS, Eder JP, et al. Pembrolizumab for the treatment of non-small-cell lung cancer. N Engl J Med. 2015;372(21):2018-28.

11. Lim SH, Sun JM, Lee SH, Ahn JS, Park K, Ahn MJ. Pembrolizumab for the treatment of non-small cell lung cancer. Expert Opin Biol Ther. 2016;16(3): 397-406.

12. Chatterjee M, Turner DC, Felip E, Lena H, Cappuzzo F, Horn L, et al. Systemic evaluation of pembrolizumab dosing in patients with advanced non-smallcell lung cancer. Ann Oncol. 2016;27(7):1291-8.

13. Chatterjee MS, Elassaiss-Schapp J, Lindauer A, Turner DC, Sostelly A, Freshwater T, et al. Population pharmacokinetic/pharmacodynamic modeling of tumor size dynamics in pembrolizumab-treated advanced melanoma. CPT Pharmacometrics Syst Pharmacol. 2017:6(1):29-39. doi:10.1002/Psp4.12140. Epub 2016 Nov 29.
14. Centers For Disease Control and Protection. Protect patients against preventable harm from improper use of single-dose/single-use vials. 2012. Available at: http://www.cdc.gov/injectionsafety/cdcposition-singleusevial. html. Accessed 25 May 2016

15. Centers For Disease Control and Prevention. Outbreaks and patient notifications in outpatient settings, selected examples, 2010-2014. http:// www.cdc.gov/hai/settings/outpatient/outbreaks-patient-notifications.html. Accessed 5 May 2017.

16. Patnaik A, Kang SP, Rasco D, Papadopoulos KP, Elassaiss-Schaap J, Beeram $M$, et al. Phase I study of pembrolizumab (Mk-3475; anti-Pd-1 monoclonal antibody) in patients with advanced solid tumors. Clin Cancer Res. 2015;21(19):4286-93.

17. Elassaiss-Schaap J, Rossenu S, Lindauer A, Kang SP, De Greef R, Sachs JR, De Alwis DP. Using model-based "learn and confirm" to reveal the pharmacokinetics-pharmacodynamics relationship of pembrolizumab in the keynote-001 trial. CPT Pharmacometrics Syst Pharmacol. 2016;6:21-8. doi:10.1002/Psp4.12132.

18. Dirks NL, Meibohm B. Population pharmacokinetics of therapeutic monoclonal antibodies. Clin Pharmacokinet. 2010:49(10):633-59.

19. Keizer RJ, Huitema ADR, Schellens JHM, Beijnen JH. Clinical pharmacokinetics of therapeutic monoclonal antibodies. Clin Pharmacokinet. 2010:49(8):493-507.

20. Reck M, Rodríguez-Abreu D, Robinson AG, Hui R, Csőszi T, Fülöp A, et al. KEYNOTE-024 Investigators. Pembrolizumab versus chemotherapy for PD-L1positive non-small-cell lung cancer. N Engl J Med. 2016;375(19):1823-33. Epub 2016 Oct 8.

21. Chow LQ, Haddad R, Gupta S, Mahipal A, Mehra R, Tahara M, et al. Antitumor activity of pembrolizumab in biomarker-unselected patients with recurrent and/or metastatic head and neck squamous cell carcinoma: results from the phase lb KEYNOTE-012 expansion cohort. J Clin Oncol. 2016. [Epub ahead of print].

22. Seiwert TY, Burtness B, Mehra R, Weiss J, Berger R, Eder JP, et al. Safety and clinical activity of pembrolizumab for treatment of recurrent or metastatic squamous cell carcinoma of the head and neck (KEYNOTE-012): an openlabel, multicentre, phase 1b trial. Lancet Oncol. 2016;17(7):956-65.

23. Haddad R, Seiwert T, Pfister DG, Worden F, Liu SV, Gilbert J, et al. Pembrolizumab after progression on platinum and cetuximab in head and neck squamous cell carcinoma (HNSCC): results from KEYNOTE-055. Ann Oncol. 2016;27(suppl 6):957PD.

24. Herbst RS, Baas P, Kim DW, Felip E, Pérez-Gracia JL, Han JY, et al. Pembrolizumab versus docetaxel for previously treated, PD-L1-positive, advanced non-small-cell lung cancer (KEYNOTE-010): a randomised controlled trial. Lancet. 2016;387(10027):1540-50.

\section{Submit your next manuscript to BioMed Central and we will help you at every step:}

- We accept pre-submission inquiries

- Our selector tool helps you to find the most relevant journal

- We provide round the clock customer support

- Convenient online submission

- Thorough peer review

- Inclusion in PubMed and all major indexing services

- Maximum visibility for your research

Submit your manuscript at www.biomedcentral.com/submit
Biomed Central 